\title{
COMPORTAMIENTO DE METAMATERIAL (LHM) Y CONVENCIONAL (RHM) EN NANOESTRUCTURAS CILÍNDRICAS (NANOTUBOS)
}

\section{METAMATERIAL (LHM) AND CONVENTIONAL (RHM) BEHAVIOR OF CYLINDRICAL NANOSTRUCTURES (NANOTUBES)}

\author{
María Ester Onell ${ }^{1}$ \\ Recibido 12 de junio de 2008, aceptado 16 de junio de 2009 \\ Received: June 12, 2008 Accepted: June 16, 2009
}

\begin{abstract}
RESUMEN
En este trabajo se estudia el comportamiento convencional o "Right Handed Materials" (RHM) y el comportamiento de metamaterial o "Left Handed Materials" (LHM) desde el punto de vista clásico, en nanoestructuras cilíndricas (nanotubos) construidas imponiendo condiciones de borde a una red bidimensional infinita de circuitos LC acoplados con interacción a primeros vecinos. Tipificaremos los materiales considerando el signo del coseno del ángulo formado por los vectores velocidad de grupo y velocidad de fase, siendo metamaterial o LHM cuando el coseno del ángulo sea negativo y convencional o RHM cuando el valor del coseno sea positivo. El eje de los nanocilindros se hace coincidir, como primer caso, con la línea de transmisión dual, y como segundo caso, con la línea de transmisión directa. Este estudio muestra que ambos nanocilindros tienen un comportamiento de RHM y LHM, y además se comportan como filtros pasa alto y pasa bajo, pero ahora aparece un número discreto de frecuencias de corte en cada caso, a diferencia de lo que ocurre en las líneas de transmisión.
\end{abstract}

Palabras clave: Metamateriales, nanoestructuras cilíndricas, red bidimensional, líneas de transmisión.

\begin{abstract}
This paper studies the behavior of conventional or "Right Handed Materials" (RHM) and "Left Handed Materials" (LHM) also named metamaterial, of cylindrical nanostructures (nanotubes) from the classical point of view. The nanotubes are building imposing boundary conditions in an infinite two-dimensional network of coupled LC circuits with interaction to nearest-neighbors. In this article, materials are classified considering the sign of the cosine of the angle between the group velocity vector and the phase velocity vector, in such a way that we have LHM behavior for negative cosine and we have RHM when the cosine is positive. The axis of the nanocylinders coincides, as the first case, with the dual transmission line, and as a second case, with the direct or conventional transmission line. This study shows that both nanocyinders have RHM and LHM behavior. In addition, it is found that these nanocylinders continues to have low-pass and high-pass filter behavior, but now a discrete number of cutting frequencies appear in each case, in contrast with the transmission lines behavior.
\end{abstract}

Keywords: Metamaterials, cylindrical nanoestructures, two-dimensional network, transmission lines.

\section{INTRODUCCIÓN}

El término "metamaterial" se asocia a una estructura o medio artificial que, en un determinado rango de frecuencias, presenta propiedades electromagnéticas singulares con potencial para una gran variedad de aplicaciones (ópticas) y de microondas, tales como nuevos tipos de moduladores, banda de filtros de transición, lentes, acopladores de microondas y antenas aleatorias. En la última década, las características electromagnéticas de los metamateriales "zurdos" (LHMs, Left Handed Materials), nombre que se da a los materiales con permitividad eléctrica $\varepsilon$ y permeabilidad magnética $\mu$ negativas, se han utilizado en el diseño de nuevas aplicaciones y dispositivos por parte de la comunidad científica [1-4]. En la literatura, metamaterial "zurdo" se entiende por un material en el que la "regla de la mano derecha" no es obedecida, lo que permite que una onda electromagnética transmita energía

1 Universidad de Tarapacá. Facultad de Ciencias. Departamento de Física. Arica, Chile. E-mail: monell@uta.cl 
(con una velocidad de grupo) en la dirección opuesta a su velocidad de fase. Los materiales convencionales (RHM), también llamados normales, son materiales naturales, que refractan la luz o la radiación electromagnética, a la derecha del haz incidente, en diferentes ángulos y velocidades, cuya constante de permitividad eléctrica $\varepsilon$ es positiva así como la constante de permeabilidad magnética $\mu$.

En este estudio consideraremos el siguiente criterio para tipificar los materiales: el comportamiento será de metamaterial o LHM cuando el coseno del ángulo formado por los vectores velocidad de grupo y velocidad de fase sea negativo y el comportamiento será convencional o RHM cuando el valor del coseno sea positivo.

En el estudio de la red bidimensional [5] se usó el criterio del índice de refracción positivo o negativo para clasificar los materiales. En el presente trabajo se obtienen los mismos resultados que en la referencia [5] usando el signo del coseno del ángulo entre los vectores velocidad de grupo y velocidad de fase como indicador del comportamiento normal o de metamaterial.

\section{RED BIDIMENSIONAL INFINITA DE CIRCUITOS LC}

Para iniciar el estudio de los nanocilindros, es importante dar a conocer primeramente las características de la red bidimensional infinita, ya que es la base en la construcción de las nanoestructuras cilíndricas. Como es sabido [5-7], la red bidimensional infinita está formada por líneas de transmisión unidimensionales directas (RHTL) en paralelo en una dirección y líneas duales (LHTL), también en paralelo, en la otra dirección.

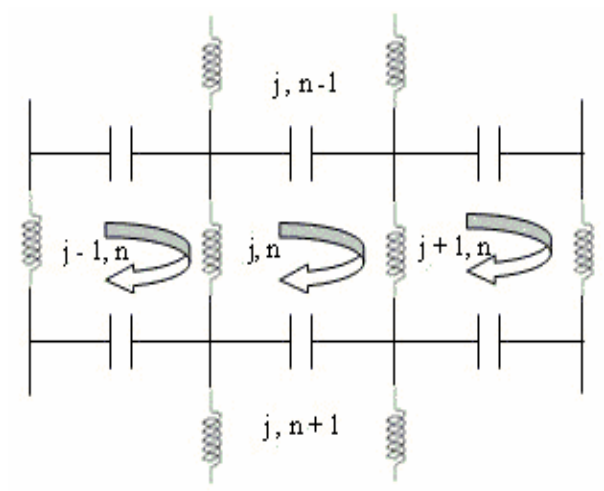

Figura 1. Red planar formada por líneas de transmisión dual en la dirección horizontal (eje x) y por líneas de transmisión directa en la dirección vertical (eje y).
En la figura 1 se muestra un dibujo representativo de la red bidimensional infinita de circuitos LC acoplados, donde todas las inductancias L son iguales, así como también las capacitancias $\mathrm{C}$ son iguales entre sí.

La red bidimensional infinita es un sistema invariante bajo traslación, y su relación de dispersión $\omega_{k_{x}, k_{y}}$ está dada por la expresión [5-7]

$$
\omega_{k_{x}, k_{y}}=\frac{1}{\sqrt{L C}}\left|\frac{\operatorname{sen}\left(\frac{k_{y}}{2}\right)}{\operatorname{sen}\left(\frac{k_{x}}{2}\right)}\right|
$$

donde los números reales $k_{x}$ y $k_{y}$ son los respectivos números de onda adimensionales en las correspondientes direcciones del plano.

La red bidimensional es un medio anisótropo ya que las velocidades de grupo y de fase nunca son colineales, quedando bien determinadas las condiciones para ondas de retroceso (LHM) o de avance (RHM), destacándose su comportamiento como metamaterial [5-6] para ciertas regiones del espacio de parámetros.

\section{NANOCILINDRO CON EL EJE EN LA DIRECCIÓN DE LA LÍNEA DUAL (NANOCILINDRO DUAL)}

Para construir los nanocilindros se aplican condiciones de borde a la red bidimensional en alguna de las dos direcciones del plano $x y$, expresándolas en función de la carga eléctrica Q de las celdas involucradas. Explícitamente, para construir el nanocilindro dual, la condición de borde que debemos imponer viene dada por

$$
Q_{l, 1}=Q_{l, M_{0}}, \quad \forall l
$$

donde los parámetros que aparecen en la condición de borde (2) se definen de la siguiente manera: $l$ es un número entero que rotula a las celdas columnas en la dirección del eje del cilindro, el cual coincide con la línea dual (eje $x$ ), y $M_{0}$ es un entero acotado de celdas filas (eje $y$ ) que fija el tamaño del perímetro del cilindro en la dirección de la línea directa.

Considerando una solución tipo ondas planas, la condición de borde (2), nos queda 


$$
Q_{0} e^{i\left(l k_{x}+k_{y}-\omega t\right)}=Q_{0} e^{i\left(l k_{x}+M_{0} k_{y}-\omega t\right)}
$$

Simplificando términos semejantes encontramos el conjunto de valores permitidos de $k_{y}$ en función del canal $n$ y del perímetro $M_{0}$

$$
\frac{k_{y}}{2}=\frac{n \pi}{M_{0}-1}=k_{0 y}, n \in \mathbb{Z}
$$

Nótese que $k_{0 y}$ es un parámetro constante.

Reemplazando (4) en (1) encontramos la relación de dispersión $\omega_{n}\left(k_{x}\right)$ para el nanocilindro dual en función del canal $n$ y del número de onda $k_{x}$

$$
\omega_{n}\left(k_{x}\right)=\frac{1}{\sqrt{L C}}\left|\frac{\sin \left(k_{0 y}\right)}{\sin \left(\frac{k_{x}}{2}\right)}\right|
$$

La figura 2 muestra la relación de dispersión en función del número de onda $k_{x}$ para los canales $n=1,2, \ldots\left(M_{0}-2\right)$. Para todos los cálculos numéricos tomaremos $M_{0}=10$, y por simplicidad haremos $L C=1$.

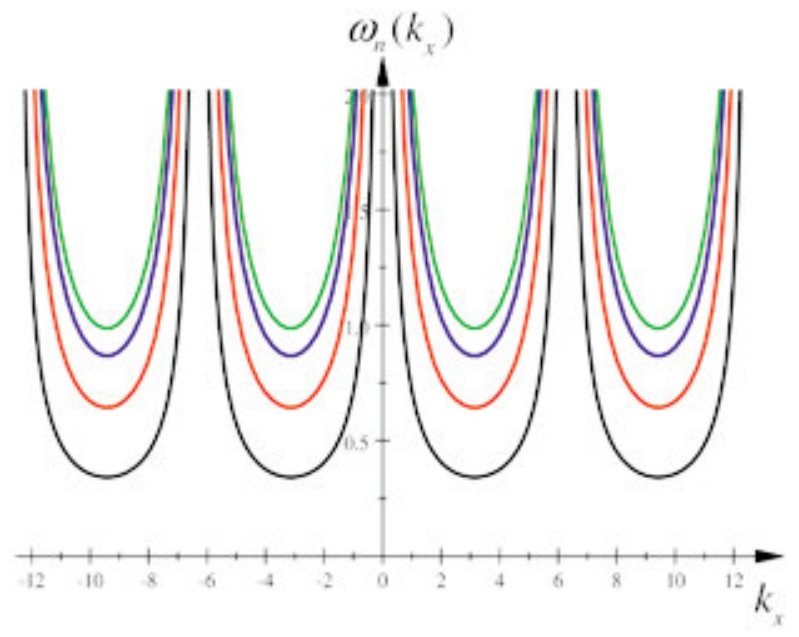

Figura 2. Relación de dispersión del nanocilindro dual en función del número de onda $k_{x}$ para los primeros $n$ canales.

La figura 2 muestra que a medida que aumenta el valor del canal $n$, la frecuencia de corte $\omega_{n}\left(k_{x}\right)$ aumenta, encontrándose que, para un perímetro $M_{0}$ dado, existe un número finito de frecuencias de corte distintas en función del canal $n$, que es igual a la parte entera de la expresión $\left(\frac{M_{0}-1}{2}\right)$, sea $N_{M_{0}}$ dicho número entero. El resto de las frecuencias coinciden con las primeras $N_{M_{0}}$. El nanocilindro dual se comporta como un filtro pasa alto, semejante a la línea de transmisión dual, solo que ahora se tiene un número $N_{M_{0}}$ de frecuencias de corte $\omega_{c}(d u a l)$ distintas dadas por $\omega_{c}($ dual $)=\frac{1}{\sqrt{L C}}\left|\sin \left(\frac{n \pi}{M_{0}-1}\right)\right|$, que van desde $\omega_{c, \min }=\frac{1}{\sqrt{L C}}\left|\sin \left(\frac{\pi}{M_{0}-1}\right)\right|$ hasta $\omega_{c, \max }=\frac{1}{\sqrt{L C}}\left|\sin \left(\frac{N_{M_{0}} \pi}{M_{0}-1}\right)\right|$

Para caracterizar el comportamiento de metamaterial del nanocilindro dual, calcularemos el coseno del ángulo que forman los vectores velocidad de fase $\vec{V}_{f}$ y velocidad de grupo $\vec{V}_{g}$, cuyas expresiones se obtienen a partir de sus respectivas definiciones, usando la relación de dispersión (5).

La velocidad de fase $\vec{V}_{f}\left(k_{x}\right)$ se propaga en las dos direcciones del plano $(x, y)$, siendo función del número de onda $k_{x}$ a lo largo del eje $x$, pero, dependiendo paramétricamente del perímetro $M_{0} \mathrm{y}$ del canal $n$ a lo largo del eje $y$,

$$
\vec{V}_{f}\left(k_{x}\right)=\frac{\omega_{n}\left(k_{x}\right)}{\left(k_{x}^{2}+k_{0 y}^{2}\right)}\left(k_{x} \hat{i}+k_{0 y} \hat{j}\right)
$$

En cambio, la velocidad de grupo $\vec{V}_{g}\left(k_{x}\right)$ sólo se propaga en la dirección del eje del nanocilindro dual, el eje $x$ en este caso, dado que la relación de dispersión (5) sólo depende del número de onda $k_{x}$, y por lo tanto su derivada parcial con respecto a $k_{y}$ es cero,

$$
\vec{V}_{g}\left(k_{x}\right)=V_{g}\left(k_{x}\right) \hat{i}=-\frac{1}{2} \omega_{n}\left(k_{x}\right) \cot \left(\frac{k_{x}}{2}\right) \hat{i}
$$


El coseno del ángulo $\gamma$ entre el vector velocidad de grupo $\vec{V}_{g}\left(k_{x}\right)$ y el vector velocidad de fase $\vec{V}_{f}\left(k_{x}\right)$, viene dado por

$$
\cos \gamma=\frac{k_{x}}{\sqrt{k_{x}^{2}+k_{0 y}^{2}}} \frac{V_{g}}{\left|V_{g}\right|}
$$

Como se dijo anteriormente, el comportamiento será de metamaterial cuando $\cos \gamma<0$, y convencional o normal cuando $\cos \gamma>0$. De acuerdo a este criterio, la figura 3 muestra las zonas donde el cilindro dual tiene comportamiento de metamaterial (LHM) y convencional (RHM), considerando distintos valores para el canal $n$

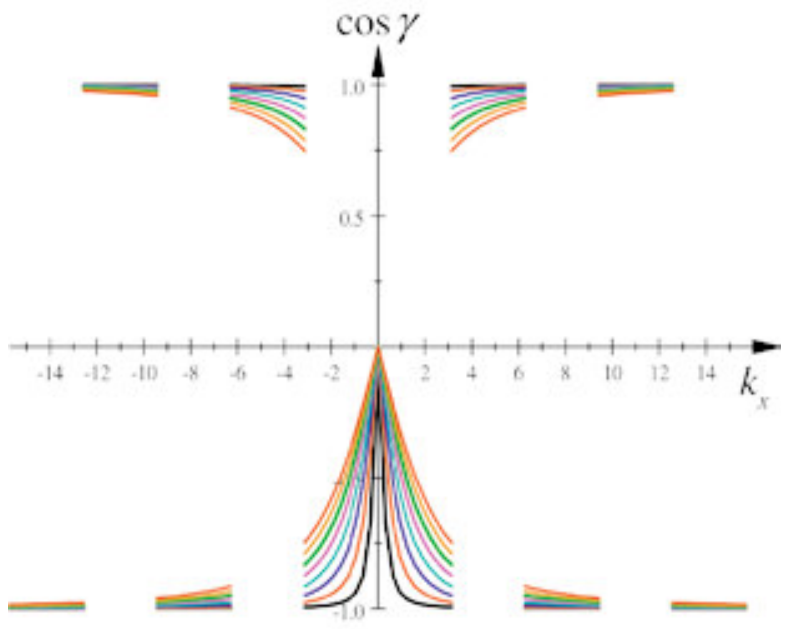

Figura 3. $\cos \gamma$ en función del número de onda $k_{x}$ para el nanocilindro dual.

Nótese que el $\cos \gamma$ tiene valores distintos para cada valor del canal $n$, a diferencia de la relación de dispersión (5) la cual sólo tiene $N_{M_{0}}$ curvas distintas.

La figura 3 muestra que en la zona comprendida entre $-\pi$ y $\pi$, el sistema tiene un comportamiento de metamaterial para cualquier valor de $n$. Además se ve claramente, en esta misma zona, que las curvas se alejan del valor -1 a medida que crece el valor del canal $n$. Fuera de la zona anterior, se puede ver que para valores crecientes del número de ondas $k_{x}$ las velocidades de fase y de grupo tienden a ser colineales (paralelas y antiparalelas), es decir, alternadamente existen regiones con comportamiento de metamaterial definidas por $k_{x} \in[2 l \pi,(2 l+1) \pi], l \in \mathbb{Z}, \mathrm{y}$ regiones con comportamiento normal en las regiones complementarias a la anterior. En el límite cuando $k_{y} \gg k_{0 x}\left(\frac{n \pi}{M_{0}-1}\right)$, se tiene que $\cos \gamma$ tiende a los valores \pm 1 , es decir, todas las curvas se acercan asintóticamente a las valores \pm 1 , lo que es una indicación de que las velocidades de grupo y de fase son prácticamente colineales.

\section{NANOCILINDRO CON EJE EN LA DIRECCIÓN DE LA LÍNEA DIRECTA O CONVENCIONAL (NANOCILINDRO DIRECTO)}

En este caso hacemos un desarrollo muy similar al del nanocilindro dual. Para construir este nanocilindro directo se aplican condiciones de borde a la red bidimensional infinita en la dirección del eje $y$, expresándolas en función de la carga eléctrica $Q$ de las celdas involucradas. Explícitamente, para este caso se tiene

$$
Q_{1, p}=Q_{M_{0}, p}, \quad \forall p
$$

donde los parámetros que aparecen en la condición de borde (9) se definen de la siguiente manera: $p$ es un número entero que rotula a las celdas fila en la dirección del eje del cilindro, el cual coincide con la línea directa (eje y), y $M_{0}$ es un entero acotado de celdas columna (eje $x$ ) que fija el tamaño del perímetro del cilindro en la dirección de la línea dual.

Considerando una solución tipo ondas planas para la condición de borde (9), encontramos el conjunto de valores permitidos de $k_{x}$ en función del canal $n$ y del perímetro $M_{0}$ del nanocilindro directo

$$
\frac{k_{x}}{2}=\frac{n \pi}{M_{0}-1}=k_{0 x}, n \in \mathbb{Z}
$$

Reemplazando (10) en (1) encontramos la relación de dispersión para el nanocilindro directo en función del canal $n$ y del número de onda $k_{y}$ :

$$
\omega_{n}\left(k_{y}\right)=\frac{1}{\sqrt{L C}}\left|\frac{\sin \left(\frac{k_{y}}{2}\right)}{\sin \left(k_{0 x}\right)}\right|
$$


La figura 4 muestra el comportamiento de la frecuencia $\omega_{n}\left(k_{y}\right)$ en función de $k_{y}$ para distintos valores del canal $n$. Tal como en el caso del nanocilindro dual, acá también existe sólo un número finito $N_{M_{0}}$ de valores distintos de frecuencias en función del canal $n$.

Tal como antes, $N_{M_{0}}$ es igual a la parte entera de $\left(\frac{M_{0}-1}{2}\right)$.

El resto de las frecuencias para los distintos valores de $n$ coinciden con éstas.

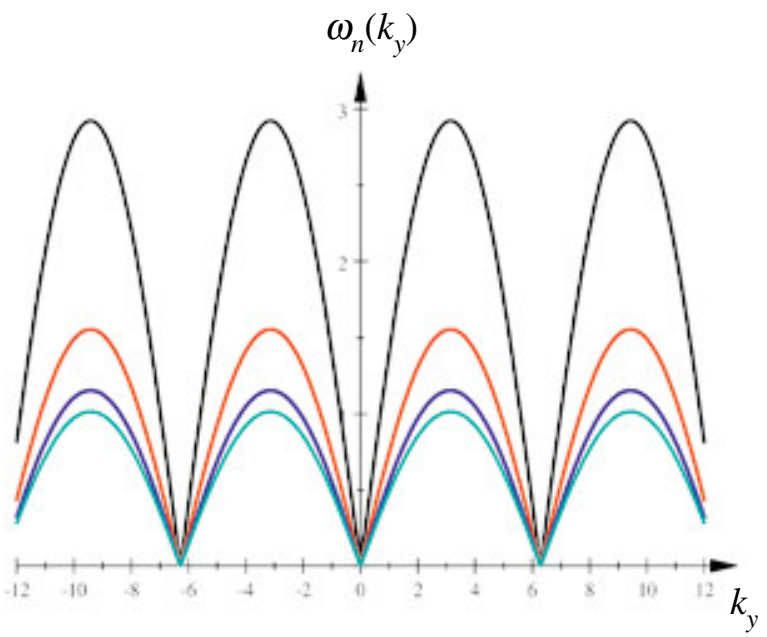

Figura 4. Relación de dispersión del nanocilindro directo en función del número de onda $k_{y}$ para los primeros $n$ canales.

El nanocilindro directo se comporta como un filtro pasa bajo, semejante a la línea de transmisión directa, solo que ahora se tiene un número $N_{M_{0}}$ de frecuencias de corte $\omega_{c}($ dir $)$ distintas dadas por

$\omega_{c}($ dir $)=\frac{1}{\sqrt{L C}}\left|\sin \left(\frac{n \pi}{M_{0}-1}\right)\right|^{-1}$, que van desde

$\omega_{c, \min }=\frac{1}{\sqrt{L C}}\left|\sin \left(\frac{N_{M_{0}} \pi}{M_{0}-1}\right)\right|^{-1}$ hasta

$\omega_{c, \max }=\frac{1}{\sqrt{L C}}\left|\left(\sin \frac{\pi}{M_{0}-1}\right)\right|^{-1}$

Para visualizar el comportamiento convencional y de metamaterial del nanocilindro directo, calculamos el coseno del ángulo $\gamma$ entre el vector velocidad de grupo $\vec{V}_{g}\left(k_{y}\right)$ y el vector velocidad fase $\vec{V}_{f}\left(k_{y}\right)$. Las velocidades de fase y de grupo para este caso se obtienen a partir de sus respectivas definiciones, usando la relación de dispersión.

La velocidad de fase $\vec{V}_{f}\left(k_{y}\right)$ se propaga en las dos direcciones del plano $(x, y)$, siendo función del número de onda $k_{y}$ a lo largo del eje $y$, pero dependiendo paramétricamente del perímetro $M_{0} \mathrm{y}$ del canal $n$ a lo largo del eje $x$,

$$
\vec{V}_{f}\left(k_{y}\right)=\frac{\omega_{n}\left(k_{y}\right)}{\left(k_{0 x}^{2}+k_{y}^{2}\right)}\left(k_{0 x} \hat{i}+k_{y} \hat{j}\right)
$$

En cambio, la velocidad de grupo $\vec{V}_{g}\left(k_{y}\right)$ sólo se propaga en la dirección del eje del nanocilindro directo, el eje $y$ en este caso, dado que la relación de dispersión (11) sólo es función de $k_{y}$, y por lo tanto su derivada parcial con respecto a $k_{x}$ vale cero,

$$
\vec{V}_{g}\left(k_{y}\right)=V_{g}\left(k_{y}\right) \hat{j}=\frac{1}{2} \omega_{n}\left(k_{y}\right) \cot \frac{k_{y}}{2} \hat{j}
$$

El coseno del ángulo $\gamma$ vale

$$
\cos \gamma=\frac{k_{y}}{\sqrt{k_{0 x}^{2}+k_{y}^{2}}} \frac{V_{g}}{\left|V_{g}\right|}
$$

La figura 5 muestra el comportamiento de $\cos \gamma$ en función del número de onda $k_{y}$.

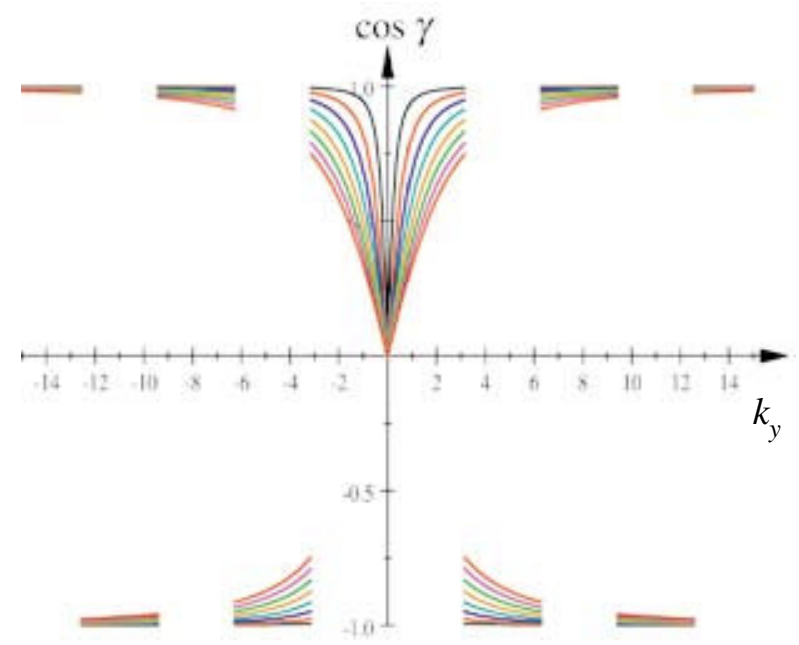

Figura 5. $\cos \gamma$ en función del número de onda $k_{y}$ para el nanocilindro directo. 
En dicha figura podemos ver que en la zona comprendida entre $-\pi$ y $\pi$, el sistema tiene un comportamiento convencional para cualquier valor de $n$. Además se ve, en esta misma zona, que las curvas se alejan del valor +1 a medida que crece el valor del canal $n$. Fuera de la zona anterior, se puede ver que para valores crecientes del número de ondas $k_{y}$ las velocidades de fase y de grupo tienden a ser colineales (paralelas y antiparalelas), mostrándose comportamientos de metamaterial y convencional. En el límite cuando $k_{y} \gg k_{0 x}\left(\frac{n \pi}{M_{0}-1}\right)$, se tiene que $\cos \gamma$ tiende a los valores \pm 1 , es decir, todas las curvas se acercan asintóticamente a las valores \pm 1 , lo cual es una indicación de que las velocidades de grupo y de fase son prácticamente colineales.

\section{ANÁLISIS DE RESULTADOS}

Uno de los resultados más importantes de este trabajo es que, dependiendo del valor fijo del perímetro $M_{0}$ de los nanocilindros, aparece un número discreto $N_{M_{0}}$ (parte entera del número $\left(\frac{M_{0}-1}{2}\right)$ ) de frecuencias de corte distintas para cada tipo de nanocilindro, dual o directo, las cuales se localizan a la izquierda y a la derecha de la frecuencia de corte de la línea directa o dual. Sin embargo, los nanocilindros dual y directo conservan el carácter de filtro paso alto y filtro paso bajo de las líneas de transmisión dual y directa.

Otro resultado importante es que, independientemente del valor del canal $n$, el nanocilindro dual presenta comportamiento de LHM en la región $k_{x} \in[2 l \pi,(2 l+1) \pi], l \in \mathbb{Z}, \quad$ y que el nanocilindro directo presenta comportamiento del mismo tipo en la región complementaria con la anterior. Además, en ambos casos, cuando se cumple la condición $k \gg\left(\frac{n \pi}{M_{0}-1}\right)$, se tiene que $\cos \gamma \rightarrow \pm 1$, es decir, todas las curvas se acercan asintóticamente a los valores \pm 1 , por lo cual se logra que las velocidades de grupo y de fase sean prácticamente colineales, comportamiento que no es posible en la red bidimensional.

Por otra parte, a diferencia de la red bidimensional, en la región comprendida entre $-\pi$ y $\pi$, estos nanocilindros se comportan sólo como material LHM para el nanotubo dual y sólo como material RHM para el nanotubo directo.
En términos aplicados, si consideramos las dimensiones de la red bidimensional $L_{x} \approx L_{y} \cong 0,1(\mathrm{~m})$ y la longitud de onda de la onda electromagnética que se propaga en el vacío $\lambda_{0}=\frac{c}{f}$, donde la frecuencia $f$ es del orden de $30 \mathrm{GHz}$, se tiene que $\lambda_{0}=0.01(\mathrm{~m})$. En el sustrato, la velocidad de la onda es $\frac{c}{\sqrt{\varepsilon_{r}}}$, siendo la permitividad relativa $\varepsilon_{r}=\frac{\varepsilon}{\varepsilon_{0}}=k$, y considerando la constante dieléctrica $k=100$, podemos determinar la longitud de onda $\lambda\left(\varepsilon_{r}\right)$ en el sustrato, cuyo valor resulta ser $\lambda\left(\varepsilon_{r}\right)=0.001(m)$. En consecuencia $\lambda\left(\varepsilon_{r}\right)<<L_{x} \approx L_{y} \cong 0.1(\mathrm{~m})$, entonces cualquier nanocilindro de tamaño finito cumple las condiciones al infinito que hemos impuesto a nuestros nanosistemas.

\section{CONCLUSIONES}

En este trabajo se han estudiado los cambios que se producen con respecto a los comportamientos de metamaterial (LHM) y convencional (RHM) de una red bidimensional infinita cuando, mediante la aplicación de condiciones de borde apropiadas, logramos transformar la red bidimensional de circuitos LC en nanocilindros de circuitos LC con su eje en la dirección de la línea dual o de la línea directa.

Una potencial aplicación de los nanocilindros estudiados en este trabajo está en la posibilidad de formar parte de aquellos dispositivos, componentes y sistemas que trabajan en el rango frecuencial de los $\mathrm{GHz}$, tales como acopladores de microondas, amplificadores, filtros, antenas y circuitos híbridos de microondas.

\section{REFERENCIAS}

[1] A. Grbic and G.V. Eleftheriades. "Negative Refraction, Growing Evanescent Waves and SubDiffraction Imaging in Loaded - Transmission - Line Metamaterials". Trans. on Microwave Theory and Techniques. Vol. $51 \mathrm{~N}^{\mathrm{o}} 12$, pp. 2297-2305. 2003.

[2] C. Caloz and T. Itoh. "Electromagnetic Metamaterials: Transmission line theory and microwave". John Wiley \& Sons. Inc. Publication. 2006.

[3] G.V. Eleftheriades, A. K. Iyer and P. C. Kremer. "Planar negative refractive index media using 
periodically L-C loaded transmission lines". IEEE Trans. Microwave Theory Tech. Vol. $50 \mathrm{~N}^{\circ} 12$, pp. 2702-2712. 2002.

[4] H. Torres-Silva. "Chiral Waves in a Metamaterial Medium”. Ingeniare. Revista chilena de ingeniería. Vol. 16 número especial, pp.119-122. 2008. ISSN 0718-3305. URLs: http://www.scielo.cl/pdf/ ingeniare/v16nespecial/art17.pdf

[5] B. Glass. "Red bidimensional de circuitos cuánticos". Tesis para optar al grado de Magíster en Ciencias con mención en Física. Universidad de Tarapacá. 2007.
[6] B. Glass. "Waves in 2D anisotropic L-C lattice metamaterials: phenomenology and properties". Microwaves and Optical Technology Letters. Vol. $50 \mathrm{~N}^{\circ}$ 10, pp. 2730-2732. 2008.

[7] M.E. Onell. "Espectro energético de una red bidimensional de circuitos LC acoplados y propiedades térmicas de nanoestructuras cilíndricas". Tesis para optar al grado de Magíster en Ciencias con mención en Física. Universidad de Tarapacá. 2007. 